\title{
Optimum Scheduling to Mitigate Inter-Wireless Body Area Network's Interference
}

\author{
Laila Nassef $^{1,2}$, Rasha Almarshdi ${ }^{1}$ \\ Department of Computer Science, Faculty of Computing and Information Technology, King Abdulaziz University, \\ Jeddah, Saudi Arabia ${ }^{1}$ \\ Department of Computer Sciences and Information, Institute of Statistical Studies and Research, Cairo University, \\ Egypt $^{2}$
}

\begin{abstract}
Wireless Body Area Network (WBAN) consists of various biomedical sensors to monitor different vital signs of patient's health. MAC layer is responsible for scheduling data transmissions, and coordinating nodes' channel access that avoids an possible collisions during data transmissions. The coexistence of multiple WBANs introduce interferences and increases the probability of collisions. A cognitive MAC, which utilizes the concept of cognitive radio, is proposed to optimally assign unique timeslots to interfered sensor nodes using Hungarian minimum matching algorithm to mitigate inter-WBAN interference. The performance of the proposed protocol is evaluated, analyzed, and compared with the standard MAC protocol using different performance metrics and scenarios. From the simulation results, we show the effectiveness of our proposed protocol in terms of throughput, latency, and energy consumption.
\end{abstract}

Keywords: WBAN; IEEE 802.15.4; MAC; Inter-Interference Mitigation

\section{INTRODUCTION}

The interest in using Wireless Body Area Network (WBAN) for medical and non-medical applications has grown significantly to improve the quality of life. A WBAN consists of a number of biomedical sensor nodes to monitor physiological data such as temperature, blood pressure, electrocardiography (ECG), electroencephalography (EEG), electromyography, and heart rate [1]. The coordinator controls the network and allocates transmission timeslots to the monitored vital signs. The efficient slot allocation is a challenging task due to the resource constraint involved in WBAN such as limited energy, processing power, storage and transmission capability. IEEE 802.15.4 [2] is currently being used as a standard for WBANs. It defines the physical (PHY) layer and medium access control (MAC) layer specifications. It operates in unlicensed $2.4 \mathrm{GHz}$ Industrial, Scientific and Medical (ISM) frequency band. IEEE802.15.4 does not specify any mechanism to mitigate interference caused by other WBANs that coexist in the same area and use the same technology [3].

On the other hand, IEEE 802.15.6 standard proposed in 2012 [ 4] divided coexistence environment into dynamic, semi-dynamic, and static conditions according to the coexisting WBANs mobility. Also, it defined three different coexistence mitigation schemes: beacon shifting, channel hopping, and active superframe interleaving to handle the coexistence problem [5]. However, IEEE 802.15.6 does not specify detailed algorithms for these coexistence mitigation schemes. Therefore IEEE802.15.4 will continue to be used and urgently need algorithms to mitigate interference.

The coexistence of multiple WBANs may cause interference and loss of monitoring data which may lead to a dangerous life treating situation [6]. In general, interference categorized into three types [7]. The first is intra-WBAN interference which occurs among sensor nodes in the same network. The second is inter-WBAN interference occurs among WBANs working at the same frequency band. The third is cross-interference caused by other technologies transmitting in the same band. This paper focuses on the second type of interference.

Cognitive Radio (CR) [8] is next-generation wireless communications, and artificial intelligence has been widely adopted to provide self-learning to observe, learn and take action against its operating environment. The application of these cognitive concepts in WBAN's environment can help to solve inter-WBAN interference. Many of CR based MAC protocols have been developed separately for Cognitive Radio Network (CRN) and Cognitive Radio Sensor Network (CRSN), but very few CR MAC protocols have been proposed to mitigate interference in WBAN. Therefore, a MAC protocol based on CR cycle will be proposed to sense, decide, and adapt scheduling of WBAN's nodes to mitigate the impact of interference. The performance of the proposed protocol will be simulated, evaluated, analyzed, and compared with the basic MAC protocol.

This paper organized as follows: Section II describes currently proposed MAC solutions to mitigate interference. The proposed cognitive MAC protocol with an analytical model are described in Section III, where the Hungarian minimum 
weight matching algorithm is used to optimally assign unique timeslots to interfered nodes. The simulation of WBANs is described in Section IV wherein a realistic channel model is used. The simulation measure performance using throughput, latency, and energy consumption metrics in various simulation scenarios of varying traffic loads and transmission power. Section V describes the conclusion and future work.

\section{RELATED WORKS}

MAC layer has four frame types which are beacon frames, data frames, acknowledgment frames, and MAC control frames. The superframe structure that defined by the coordinator divided into an active and inactive period as shown in Fig. 1. The active period is divided into Contention Access Period (CAP) with nine timeslots and Contention Free Period (CFP) with seven timeslots. Two transmission modes are supported: a beacon-enabled mode and non beaconenabled mode [9]. In beacon-enabled mode, the beacon is sent by the coordinator in the first slot of each superframe to synchronize the attached nodes. The CAP is accessed using slotted Carrier Sense Multiple Access with Collision Avoidance (CSMA/CA). The CFP provides Guaranteed Time Slot (GTS) to support QoS requirements of applications [10].

The time between two consecutive beacon frames is defined as the Beacon Interval (BI), which depends on the data rate. The Superframe Duration (SD) defines the active portion in the BI and it is divided into 16 timeslots with the same length. BI and SD are determined by Beacon Order (BO) and Superframe Order (SO) [11].

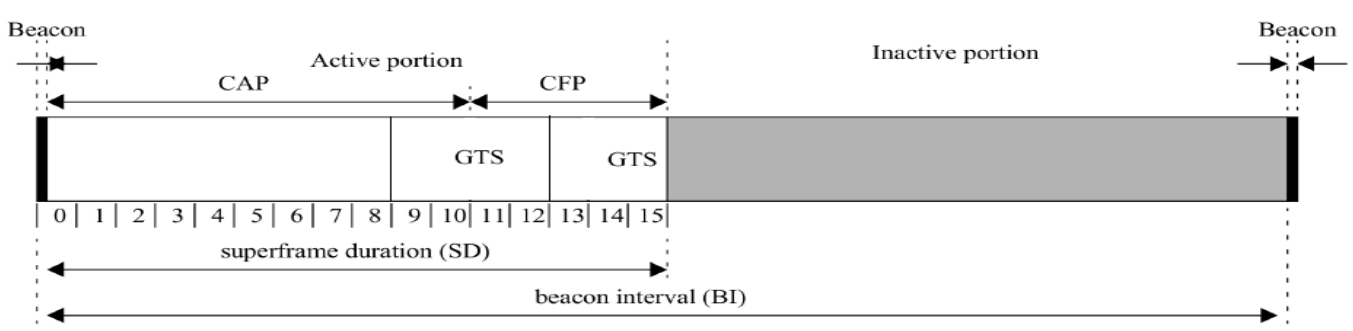

Fig. 1: IEEE 802.15.4 superframe structure

There are numbers of MAC protocols have been proposed to mitigate inter-WBAN interference in IEEE 802.15.4. A decentralized interference mitigation scheme (DIM) is proposed in [12] where a hybrid scheduling technique that combines the CSMA/CA and TDMA mechanisms are used. The DIM superframe consists of a beacon, scheduling phase (SP) that accessed using GTS, and CAP. The SP is scheduled dynamically according to interference. The DIM scheme doesn't require collaboration between the coexistence WBANs to mitigate interference, but it consumes high energy and high overhead. A fairness-based throughput maximization heuristic (FTMH) algorithm is proposed in [13] where it uses the heuristic technique that solve the problem in a global solution when the classical method fail. The FTMH uses non-linear programming and fairness in medium access to mitigate inter-WBAN interference. The superframe divided into multiple timeslots and only one sensor node is assign one timeslot for data transmission. The ratio of successful access for the sensor node in the time slot is calculated. The sensor node with the highest success ratio is assigned the timeslot with the highest interference. This algorithm aims to maximize the network throughput, but the interference is partially mitigated.

Few MAC protocols have used the CR concept. A fast dynamic cognitive radio (FDCR) algorithm is proposed in [14] to minimize the impact of co-channel interference that comes from other WBANs and provides resource allocation. FDCR algorithm performs two phases. First, channel sensing phase where the coordinator senses the spectrum to detect the interference using Received Signal Strength Indicator (RSSI) and determine the channel statue if ideal or not. Second, channel access phase where the coordinator evaluates the channel condition. If the channel in the ideal state, the coordinator sends to its sensor nodes to access the channel. This algorithm is effective to mitigate inter-WBAN interference, but the latency is high when the beacon collision occurs. An interference-aware management framework (IAMF) scheme with cognitive radio attached to each sensor node is proposed in [15] to avoid interference. The IAMF scheme detects the regions of interference among WBANs and calculates the position of sensor nodes. It performs two tasks to avoid interference which is power control and frequency hopping. A cognitive-receiver initiated cyclEd receiver (C-RICER) protocol was proposed in [16] to mitigate the highest level of interference based on three cognitive tasks which are channel sensing, power adaption, and channel adaption. Same FDCR, the coordinator senses the channels in the network using RSSI to build the interference map. The power adaption used to reduce energy consumption caused by interference where the channel adaption used by the coordinator to switch to another channel if the interference level is more than threshold value. C-RICER is more efficient than FDCR and IAMF, but the interference map construction leads to the packet delay. 
From the previous work presented in the literature, there is no protocol mitigates inter-WBAN interference completely taking into account the reduction of energy consumption and latency. So, a cognitive MAC protocol to mitigate interWBAN interference will be proposed where the coordinator will have CR capabilities to cooperate with other coordinators to mitigate inter-WBAN interference. The coordinator will sense the channel state, identify the interfered nodes, and then adapt scheduling to optimize network performance with low energy consumption and latency.

\section{III.PROPOSED COGNITIVE MAC PROTOCOL}

The proposed cognitive MAC protocol that aims to mitigate inter-WBAN interference is based on the CR cycle of sense, decide, and adapt. The topology of isolated independent WBANs is assumed to be a star topology that uses the beacon-enabled mode. When interference occurs the coordinators cooperate to adapt transmission and to switch to the newly assigned timeslots.

\section{A. Network Model}

The coexisting WBAN denoted by $\left\{W_{B A N} \mid k=1, \ldots, N\right\}$, Where $\mathrm{k}$ is the number of WBAN. Each WBAN consist of one coordinator and $\mathrm{M}$ sensor node. For intra-body communication which occurs between the coordinator and sensors in the same WBAN, the transmission range denoted as a circle. Inter-body communication defined as the communication between the coordinators of multiple WBANs in the same frequency band. If there is a node or multiple nodes from other WBAN in the transmission range of a node as shown in Fig. 2, inter-WBAN interference occurs, and these nodes are called interfering nodes. The proposed MAC works in three phases: channel sensing, scheduling decision, and switching phase.

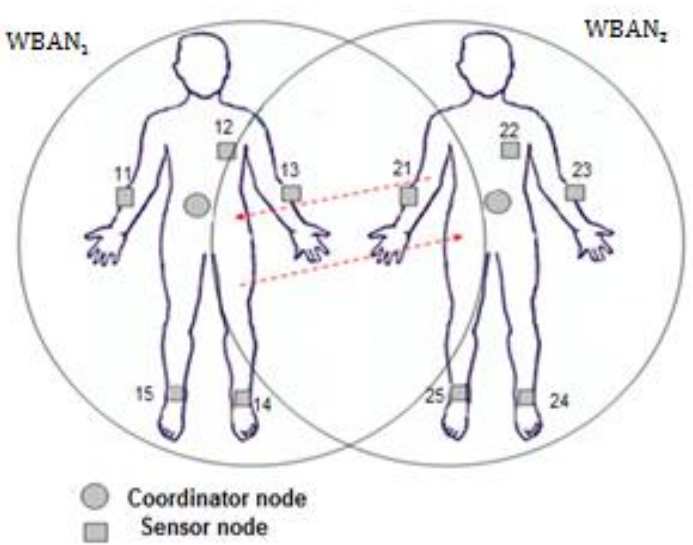

Fig. 2: The network model

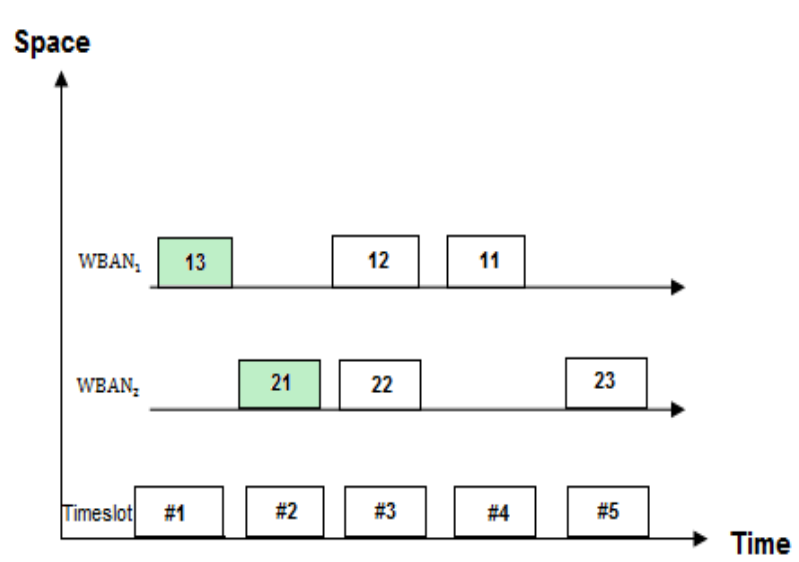

Fig. 3: Unique timeslot allocation

\section{Channel Sensing Phase:}

The coordinator is responsible for channel sensing to monitor Signal to Interference Noise Ratio (SINR) periodically to measure the interference level for each sensor node. SINR is defined as the power of a certain signal divided by the sum of the interference power (from all the other interfering signals) and the power of background noise. If the power of noise is zero, then the SINR reduces to the Signal-to-Interference Ratio (SIR). Conversely, zero interference reduces the SINR to the Signal-to-Noise Ratio (SNR) which is the bandwidth or the channel capacity of a communication channel. A ratio higher than 1 (greater than $0 \mathrm{~dB}$ ) indicates more signal than noise. The minimum threshold of RSSI value required to overcome the effect of interference is $R_{\text {thresold }}$. Each WBAN can be described by a list of sensors each with traffic priority. The transmission time of each sensor node is ratio between the packet length and the data rate priority. In this research, all nodes are assumed to have same data rate priority.

In non-interfered case, when a coordinator transmits to its sensor nodes using transmission power of $P_{c}$, this signal will reach that nodes with signal strength that can be represented as $A=\frac{P_{c}}{d(c, s)^{\beta}}$ where $\beta$ is the path loss exponent of the channel and $d(c, s)$ represent the Euclidean distance between them. Knowing that direct communication between two nodes is only possible if the $d(c, s) \leq R_{t x}$, where $R_{t x}$ is the transmission range, thus nodes will interfere if interference range $\left(R_{\text {int }}\right)$ will be greater than transmission range i.e. $R_{\text {int }}>R_{t x}$.

Consequently, in interfered case, the transmission from other WBAN's nodes will cause interference to that network if they operates on the same channel and they are within interference range of other nodes. The SINR for any receiving 
node will be the ratio between signal power $P_{c}$ to the combination of the background noise interference and interference from other nodes in networks. When SINR value is less than a threshold value $R_{\text {thresold }}$, then this will mean that both WBAN schedules have overlapped.

\section{Scheduling Decision Phase:}

In multiple WBANs, interference from other networks is received at sensors in interference region. Then SINR can be redefined as

$$
\gamma_{c, s}=\frac{p_{c, s}}{\sigma^{2}+\Sigma_{l \neq s} p_{l, s}}
$$

where $p_{c, s}$ is the received signal power at sensors in interference region (originates from its coordinator), $p_{l, s}$ is the interference power that originates from other WBANs (from the coordinator or any sensor) and received by the sensor node of that WBAN, and $\sigma^{2}$ is the power of additive noise.

If $\left(\gamma_{c, s}\right)<\left(\gamma_{t h}\right)$, sensor node will be added to the interfered sensor list $I S L$ from the coordinator where $I S L=\left\{\right.$ node $\left._{c, s} \mid\left(\gamma_{c, s}\right)<\left(\gamma_{t h}\right)\right\}$ where, $1 \leq s \leq M$. Otherwise, sensor node is added to the non-interfered sensor list NISL where $\left(\left(\gamma_{c, s}\right) \geq\left(\gamma_{t h}\right)\right)$.

The weighted cost function can be formulated based on many factors, but this research consider interference only. Therefore, cost matrix $C_{i, j}$ is defined as

$$
\mathrm{C}_{\mathrm{i}, \mathrm{j}}=\frac{\gamma_{i, j}}{\gamma_{t h}}, j \in I S L_{i}
$$

This function represent the cost of assigning a time slot $\mathrm{j}$ to a sensor node $\mathrm{i}$ in the ISL. Hungarian minimum weight matching algorithm will be used to solve the assignment problem to obtain optimized schedule. The main object of this algorithm is to assign non orthogonal timeslots to interfered nodes. Using this constraint, any node must be assigned only one timeslot and any timeslot must only be assigned to one node. The algorithm can be represented mathematically as:

minimize $\sum_{i=1}^{N} \sum_{j=1}^{N} C_{i, j} X_{i, j}$

Subject to

$\sum_{j=1} \quad X_{i, j}=1, \quad \forall i \in \overline{1, N}$ one node to one timeslot assignment

$\sum_{i=1} \quad X_{i, j}=1, \forall j \in \overline{1, N}$ one timeslot to one node assignment

Where $X_{i, j}$ is a binary matrix $\{0,1\}$.

\section{Switching Phase:}

The coordinators may not have any severe power constraints and is a computationally powerful device. Thus, CR capabilities are added to the coordinators to decide to maximize the shared timeslots among WBANs. The transmission number in $\mathrm{N}$ WBANs is $\mathrm{M} \times \mathrm{N}$, and $|\mathrm{T}|$ is the set of timeslots in one superframe. In each timeslot, there are up to $\mathrm{M}$ transmissions in N WBANs that go from the sensor nodes to their coordinators. To find the maximum number of WBANs that can transmit in one timeslot, the scheduling problem is formulated as an optimization problem to maximize the number of synchronous transmissions in multiple WBANs in each timeslot. The formulated optimization problem has combinatorial complexity, because of the need to test all the possible combinations of decision variables to get optimal solutions. By using the Hungarian minimum weight matching algorithm, which is a well-known combinatorial optimization method, the optimal assignment for a given cost matrix is founded.

A perfect matching between interfering nodes and transmission slots is achieved when no more than one node is allowed to switch the same timeslot in the same transmission round. The algorithm is described in Pseudocode 1. The Hungarian minimum weight matching algorithm optimally reschedule the nodes (i.e., no two sensor nodes are allowed to assign with the same timeslot in the same transmission round) to achieve interference-free scheduling (i.e., interfered nodes receive disjoint sets of timeslots as shown in Fig. 3). 


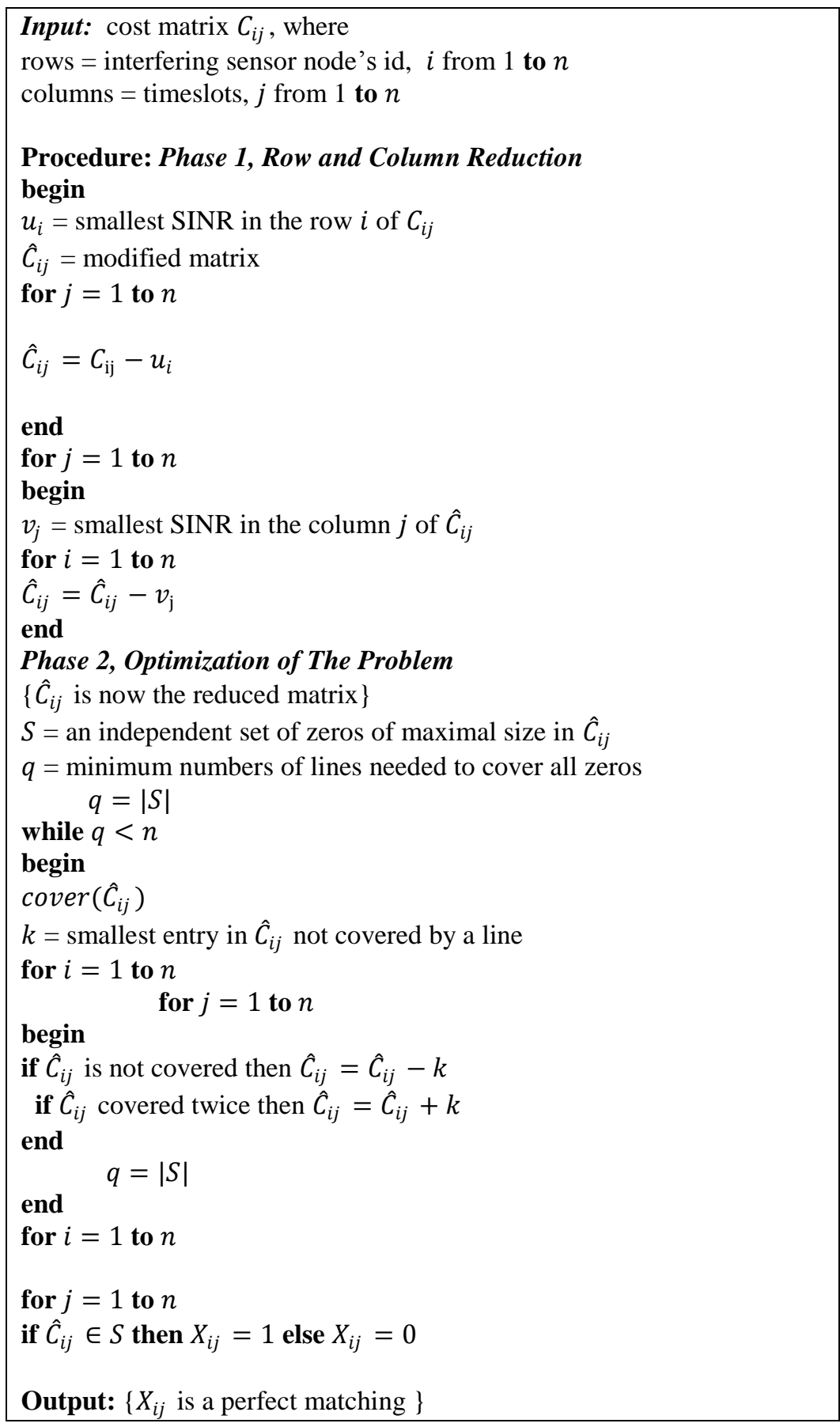

Pseudocode 1: Hungarian minimum weight matching algorithm

\section{PERFORMANCE EVALUATION}

The performance of the proposed protocol is simulated using Castalia simulator version 3.2 based on OMNET++ platform [17]. The performance of basic IEEE 802.15.4 is compared with the proposed protocol in terms of throughput, latency, and energy consumption. For simplicity, a static star topology with one coordinator in the center is modeled as a circle of the radius with $\mathrm{R}_{\mathrm{t}}$ transmission range. The sensor nodes are uniformly located at random within the circle. The number of sensors nodes are five and simulation area is $10 \mathrm{~m} \times 10 \mathrm{~m}$.

The main drawback of Castalia is that it lacks support for coexistence of multiple WBANs. On the other hand, Castalia is an open-source network simulator, and extra features can be added. Therefore, Castalia has been extended to mimic interference from other WNANs, and some modifications were made to the existing physical layer model of this simulator. For every node in the interference region, when the node generates a packet in the application layer, the transmitted signal is added to a constant source of noise to mimic the effect of coexistence with multiple WBANs. This 
means that packets sent by nodes in the interference region are considered as external noise. In this case, the interference takes place only at the start of the simulation, and the coordinator reacts by calling the rescheduling phase. The common simulation parameters are given in TABLE 1.

TABLE 1 SIMULATION PARAMETERS

\begin{tabular}{|c|c|}
\hline \multicolumn{2}{|c|}{ Application Layer Parameters } \\
\hline Packet Inter-arrival Time & $1 \mathrm{~s}$ \\
\hline Start-up Delay & $5 \mathrm{~s}$ \\
\hline Data Payload & 64 bytes \\
\hline IEEE 802.15.4 MAC Parameters \\
\hline Max Frame Trials & 10 \\
\hline Max Lost Beacons & 4 \\
\hline Packet Validity Time (Expiration date) & $8 * 122.88=983.04 \mathrm{~ms}$ \\
\hline Frame Order & $61.44 \mathrm{~ms}$ \\
\hline Beacon Order & $122.88 \mathrm{~ms}$ \\
\hline Beacon Time Out & $3 \mathrm{~ms}$ \\
\hline Buffer Size & 32 \\
\hline IEEE 802.15.4 Physical Parameters \\
\hline Transmission Power & $-25 \mathrm{dBm}$ \\
\hline Data Rate & $250 \mathrm{kbps}$ \\
\hline
\end{tabular}

IEEE 802.15.4-compliant ChipCon CC2420 transceiver [18] for the energy consumption model. All of the nodes in the WBAN operate in the beacon-enabled mode with packets arrive at the MAC sub-layer with an arrival rate of packets per second (packets/sec) and with a Poisson distribution. In the simulation, the arrival rate is assumed to be the same for all end nodes. All nodes have similar data priorities.

The channel between the sensors and the coordinator is modeled using a realistic lognormal distribution indicated in equation 4. The $\operatorname{PL}\left(d_{0}\right)$ is the path loss at reference distance $d_{0}(1 \mathrm{~m})$ which is set to $55 \mathrm{~dB}$, and $\beta$ is the path loss exponent which is set to 2.4. The shadowing term $\mathrm{X}_{\sigma}$ is a zero-mean Gaussian random variable is set to 4 . These values are selected to give a high impact of interference from other WBAN.

$$
[\operatorname{PL}(d)]_{\mathrm{dB}}=\left[\operatorname{PL}\left(d_{0}\right)\right]_{\mathrm{dB}}+10 \beta \log \left[\frac{\mathrm{d}}{\mathrm{d}_{0}}\right]+\left[\mathrm{X}_{\sigma}\right]_{\mathrm{dB}}
$$

\section{A. Simulation Scenarios}

To evaluate the performance, two different scenarios are used. First scenario, the performance of IEEE 802.15.4 and the proposed cognitive MAC protocol is performed with varying traffic load. The traffic load is the number of sending rate for the node in packets/sec/node. The second scenario, the performance of IEEE 802.15.4 and the proposed cognitive MAC protocol was performed with varying transmission power.

\section{B. Simulation Results and Analysis}

\section{Throughput:}

We can define the network throughput as the average of successful packets rate transferred from the sensors to the coordinator per unit time (measured in packets per second). As shown in Fig. 4, the proposed cognitive MAC protocol outperforms IEEE 802.15.4 in both scenarios. As in Fig. 4 (a), the perfect reception when all nodes send the packets for 50 seconds and reach 600 packets/node for 20 sending rate in IEEE 802.15 .4 and 900 packets/node for 20 sending rate in the proposed protocol. As in Fig. 4 (b), the perfect reception when all nodes send the packets in $-10 \mathrm{dBm}$ and reach 250 packets/node for the proposed protocol and 210 packets/node for IEEE 802.15.4, and this means that if the transmission power increases, the packets reception will be increase. Our proposed protocol allows for multiple WBANs to transmit at the same time using the new scheduled superframe which leads to increasing the throughput for the proposed protocol. 


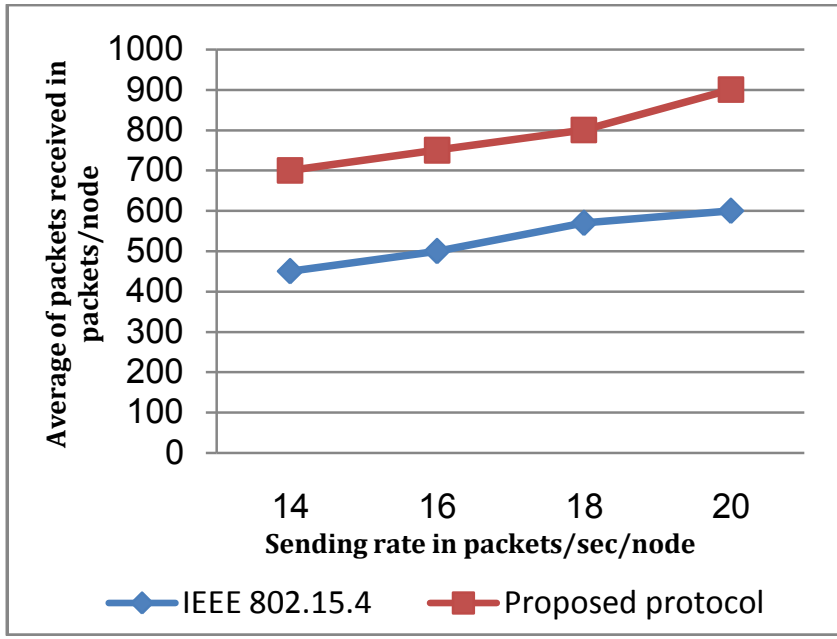

(a)

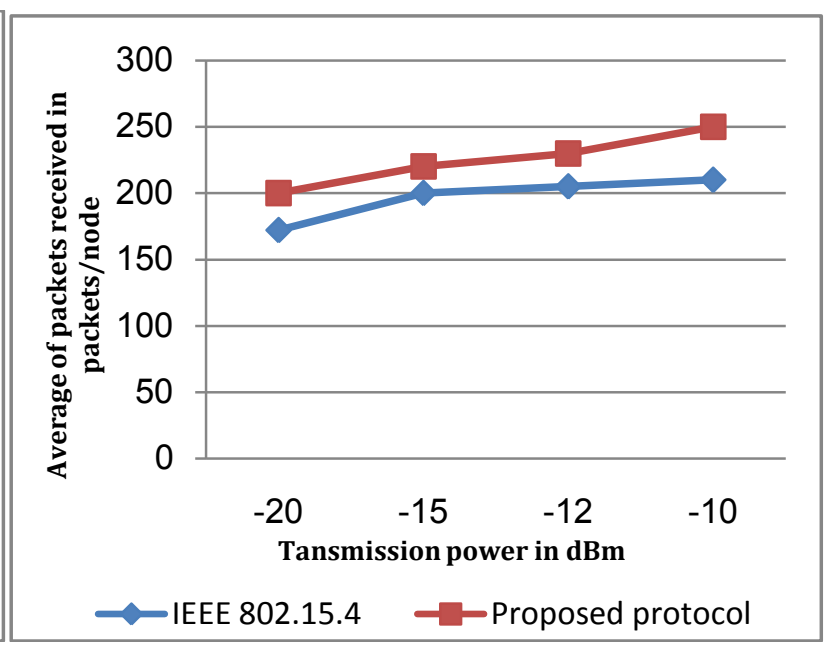

(b)

Fig. 4: Throughput for (a) varying in traffic load; (b) varying in transmission power

\section{Latency:}

The latency defined as the total time it takes for sending the packet from the sensor to the coordinator. The results of latency test are shown in Fig. 5. The latency for our proposed protocol is higher than IEEE 802.15.4 when we vary the sending rate because it takes time for beacon transmission to synchronize the interfering nodes. In the case of varying in transmission power, our proposed protocol achieves lower latency.

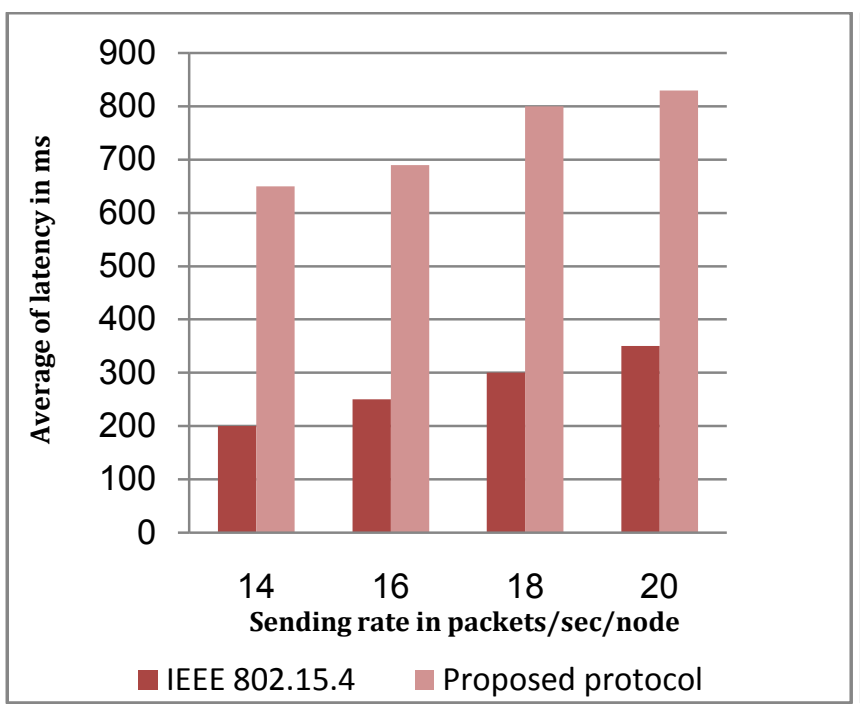

(a)

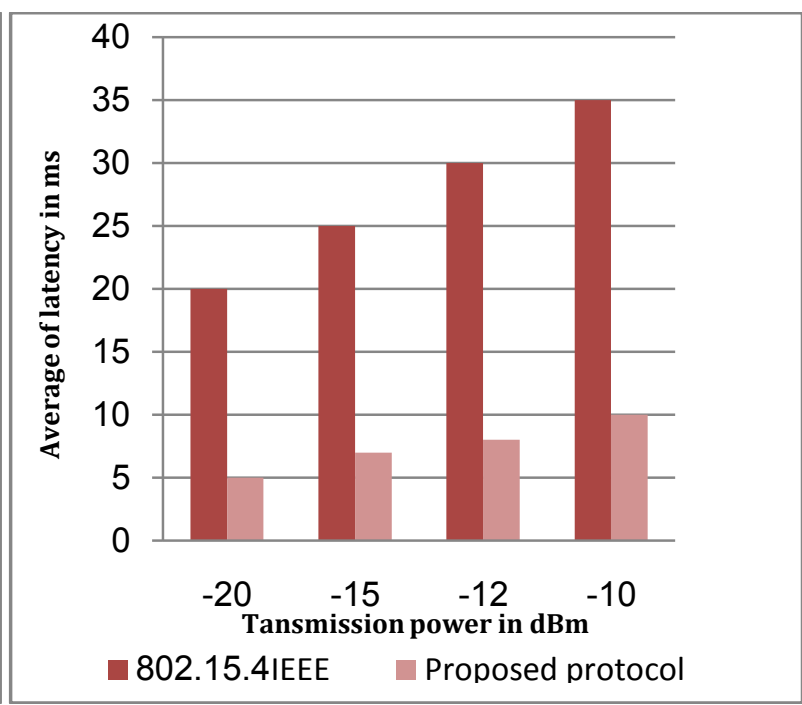

(b)

Fig. 5: Latency for (a) varying in traffic load; (b) varying in transmission power

\section{Energy Consumption:}

Energy consumption calculated in Joules per bit. Because the coordinator has low energy constraint than sensors, we focus on the energy consumed by sensors. The sensors consume energy in transmitting and receiving state. As shown in Fig. 6 (a), (b), the energy consumption of the proposed protocol lower than IEEE 802.15.4 in both scenarios. The main reason for energy consumption in the interference environment is when the packets collide due to the lack of an efficient scheduling algorithm. Our proposed scheduling algorithm has proved to be effective in reducing energy consumption with varying in sending rate and transmission power. 


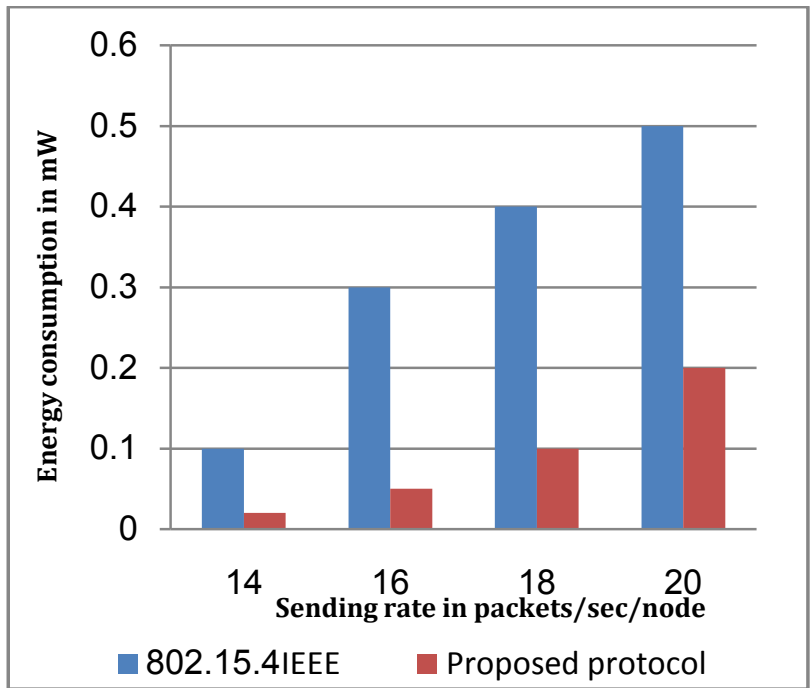

(a)

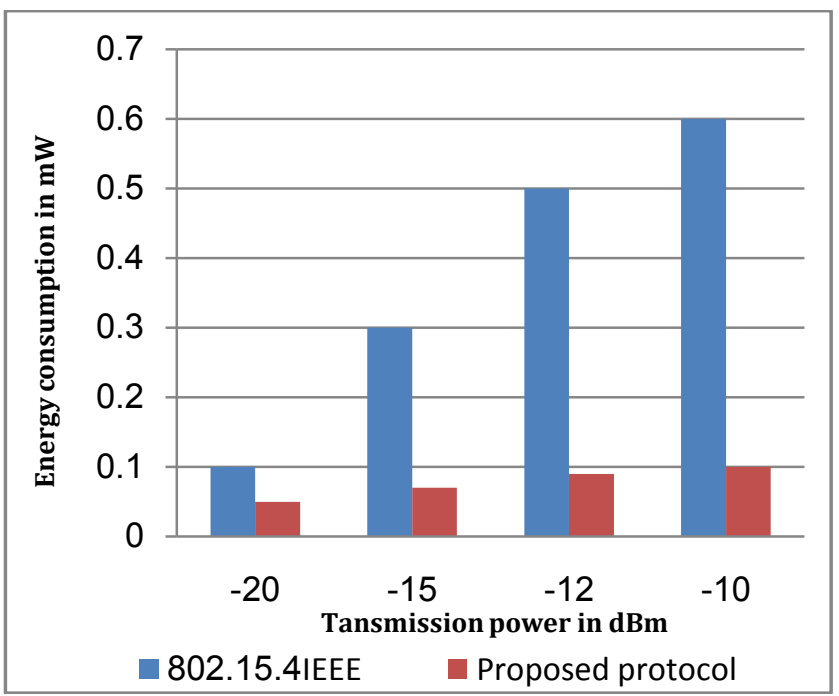

(b)

Fig. 6: Energy consumption for varying in traffic load; (b) varying in transmission power

\section{CONCLUSION}

This paper proposed a cognitive MAC protocol based on IEEE 802.15.4 standard to mitigate inter-WBAN interference. An adaptive TDMA scheduling algorithm was proposed to mitigate interference using Hungarian minimum weight matching algorithm. In our proposed protocol, three phases are performed in order to mitigate interference. Channel sensing, scheduling decision, and switching phase. The nodes that cause interference are assigned to the timeslots where the perfect matching when no more than one node assign the same timeslot in the same round. We compare between the proposed protocol with the standard protocol. We first determine the appropriate performance metrics that used in the simulation. Results analysis show that our proposed protocol proved its effectiveness and performance improvement in the term of throughput, latency, and energy consumption. As a future work, we aim to extend this work to be applied to IEEE 802.15.6 MAC protocol.

\section{REFERENCES}

[1] M. Arefin, M. Ali and A. Haque, "Wireless Body Area Network: An Overview and Various Applications", Journal of Computer and Communications, vol. 05 , no. 07 , pp. 53-64, 2017.

[2] R. Negra, I. Jemili and A. Belghith, "Wireless Body Area Networks: Applications and Technologies", Procedia Computer Science, vol. 83, pp. 1274$1281,2016$.

[3] S. Nepal, S. Dahal and S. Shin, "Does the IEEE 802.15.4 MAC Protocol Work Well in Wireless Body Area Networks?", Journal of Advances in Computer Networks, vol. 4, no. 1, pp. 52-58, 2016

[4] J. H. Kurunathan, " Study and overview on WBAN under IEEE 802.15.6", U.Porto Journal of Engineering, vol. 1, no. 1, pp. 11-21, 2015.

[5] E. Ben Hamida and M. Alam, "Performance Evaluation of IEEE 802.15.6-based WBANs under Co-Channel Interference", International Journal of Sensor Networks, vol. 1, no. 1, p. 1, 2016.

[6] F. Martelli and R. Verdone, "Coexistence Issues for Wireless Body Area Networks at 2.45 GHz", European Wireless 2012, April 18-20, 2012, Poznan, Poland, pp. 2-7, 2012.

[7] T. Le and S. Moh, "Interference Mitigation Schemes for Wireless Body Area Sensor Networks: A Comparative Survey", Sensors, vol. 15, no. 6, pp. $13805-13838,2015$

[8] G. Ghosh, P. Das and S. Chatterjee, "A Cognitive Radio And Dynamic Spectrum Access - A Study", International Journal of Next-Generation Networks, vol. 6, no. 1, pp. 43-60, 2014.

[9] S. A. Desal, P. H. Zope and S.R. Suralkar, "A Review on IEEE 802.15.4- Standard for Wireless Body Network", International Journal of Innovative Research in Science, Engineering and Technology, vol. 2, no. 5, pp. 1477-1486, 2013.

[10] M. Khanafer, M. Guennoun and H. Mouftah, "A Survey of Beacon-Enabled IEEE 802.15.4 MAC Protocols in Wireless Sensor Networks", IEEE Communications Surveys \& Tutorials, vol. 16, no. 2, pp. 856-876, 2014.

[11] R. Garg, R. Paulus, A. K. Jaiswal and A. Ashok, "Performance Analysis of a Beacon and Non Beacon Enabled IEEE 802.15.4 Star WSNs with Different Traffic Loads", International Journal of Current Engineering and Technology, vol. 4, no. 2, pp. 987-992, 2014.

[12] B. Yuan et al. "DIM: A novel decentralized interference mitigation scheme in WBAN", IEEE International Conference on Wireless Communications \& Signal Processing (WCSP), 2015.

[13] L. Ming, J. Liu, Z. Ma, C. Yuan and B. Yuan, "Throughput optimization with fairness consideration for coexisting WBANs", Communications (ICC), IEEE International Conference. IEEE, 2015.

[14] Q. Shen et al. "Adaptive Cognitive Enhanced Platform for WBAN", In Proceeding of the IEEE/CIC International Conference on Communications in China (ICCC), pp. 739-744, 2013.

[15] D. Ouattara, M. T. Quach, F. Krief, M. A. Chalouf and H. Khalife, "Mitigating the Hospital Area Communication's Interference using Cognitive Radio Networks", In Proceeding of the IEEE $15^{\text {th }}$ International conference on E-Health Networking, Applications \& Services (Healthcom), pp. 324-248, 2013.

[16] N. Q. Nhan, M. Gautier and O. Berder, "Asynchronous MAC Protocol for Spectrum Agility in Wireless Body Area Sensor Networks", In Proceeding of the International Conference on Cognitive Radio Oriented Wireless Networks and Communications (CROWNCOM), pp. 203-208, 2014.

[17] "OMNeT++ Discrete Event Simulator - Simulation models", Omnetpp.org, 2018. [Online]. Available: https://omnetpp.org/models. [Accessed: 24- Feb2018].

[18] S. Rathore, R. Paulus, A. Jaiswal and A. Agrawal, "Analysis of QOS and Energy Consumption in IEEE 802. 15. 4/ZigBee Wireless Sensor Network", International Journal of Computer Applications, vol. 121, no. 17, pp. 40-43, 2015. 